\title{
Low temperature induces cryoinjury in mouse corneal endothelial cells by stimulating the Stk11-p53 signal pathway
}

\author{
SIJIE ZHAO ${ }^{1 *}$, XINFENG FEI $^{2 *}$, TE LIU $^{3}$ and YAN LIU ${ }^{1}$ \\ ${ }^{1}$ Department of Ophthalmology, Shanghai First People's Hospital, School of Medicine, Shanghai Jiao Tong University, \\ Shanghai 200080; ${ }^{2}$ Department of Ophthalmology, The Branch of The First People's Hospital of Shanghai, Shanghai 200081; \\ ${ }^{3}$ Basic Research Laboratory, Shanghai Geriatric Institute of Chinese Medicine, Longhua Hospital, \\ Shanghai University of Traditional Chinese Medicine, Shanghai 200031, P.R. China
}

Received October 21, 2014; Accepted July 10, 2015

DOI: $10.3892 / \mathrm{mmr} .2015 .4301$

\begin{abstract}
Cryoinjury, or injury caused by extremely low temperatures, can occur in corneal endothelial cells (CECs) and lead to visual impairment. However, the mechanism of cryoinjury in CECs is not clear. The Stk11-p53 signaling pathway regulates the proliferation and division of cells. Activity of the Stk11-p53 signaling pathway arrests the cell cycle at the G0/G1 phase and induces apoptosis. In this study, a mouse model of cryoinjury in CECs was used. Following injury, significant mouse CEC death and shedding were observed. In addition, the mRNA and protein levels of core factors from the Stk11-p53 signaling pathway (Stk11, p21 and p53) were elevated and Caspase-3 was activated following cryoinjury. In addition, chromatin immunoprecipitation revealed that Stk11 catalyzed p53 serine 15 phosphorylation, and the Stk11-p53 complex bound to the $\mathrm{p} 21$ promoter and stimulated gene transcription. Thus, the results of the present study suggest that cryoinjury leads to the damage and apoptosis of mouse CECs by activation of the Stk11-p53 signaling pathway, phosphorylation of $\mathrm{p} 53$ serine 15 and $p 21$ gene transcription.
\end{abstract}

Correspondence to: Professor Yan Liu, Department of Ophthalmology, Shanghai First People's Hospital, School of Medicine, Shanghai Jiao Tong University, 100 Haining Road, Shanghai 200080, P.R. China

E-mail: yanliu201410@126.com

Professor Te Liu, Basic Research Laboratory, Shanghai Geriatric Institute of Chinese Medicine, Longhua Hospital, Shanghai University of Traditional Chinese Medicine, 365 Xiangyang Road, Shanghai 200031, P.R. China

E-mail: teliu79@126.com

${ }^{*}$ Contributed equally

Key words: corneal endothelial cells, cryoinjury, Stk11-p53 signal pathway

\section{Introduction}

The cornea is a layer of transparent film consisting of a fibrous membrane covering the front of the eye, and while the cornea appears round from behind, it appears elliptical from the front (1-5). The cornea is divided into the following five layers, from front to back: Epithelium, lamina elastica anterior (Bowman membrane), stroma, lamina elastica posterior (Descemet membrane) and endothelium (1-5). The endothelium is a monolayer of hexagonal flat corneal endothelial cells (CECs). Its matrix layer of water molecules are discharged into the anterior chamber, and the matrix in the dehydrated state is transparent, which is key for its refractive properties (1-5). The CECs maintain the corneal structure, enable corneal refraction, provide a barrier function, maintain the osmotic pressure and guarantee normal corneal metabolism (1-5). CECs are end-stage differentiated cells that can not regenerate (1-5). Damage to a large area of CECs causes edema, corneal degeneration, corneal decompensation and sometimes blindness (1-5). There are numerous factors that can cause corneal damage, such as mechanical, irradiation, chemical and freezing injuries $(6,7)$. Injury caused by freezing of CECs is common in cold regions of the world and can affect vision (7). However, to date, the mechanism of damage caused by low temperature freezing of CECs is not clear. Skt11, also termed LKB1, is a serine/threonine protein kinase, which has been implicated in the regulation of multiple biological processes and signaling pathways (8-11). Mutation of Stk11 causes Peutz-Jeghers syndrome (8-11). A previous study reported that Stk11 was recruited directly to the p21/WAF1 promoter, as well as other p53 activated promoters, in a p53-dependent manner (9). Furthermore, Stk11 could activate the p53 and p16 pathways to arrest cell cycle progression from the G0/G1 phase to $S$ phase (8). However, it is unknown whether low-temperature freezing activates the Stk11-p53 pathway to induce the apoptosis of CECs. Therefore, a liquid nitrogen mouse model was used to investigate whether low-temperature damage of mouse CECs induces expression and activation of the Stk11-p53 signaling pathway.

\section{Materials and methods}

Animals and cryoinjury treatment. Female C57BL/6 mice ( $n=30$; age, 4-5 weeks of age) were obtained from the 
Table I. Reverse transcription-quantitative polymerase chain reaction primers.

\begin{tabular}{|c|c|c|}
\hline Gene product & Primers $\left(5^{\prime} \rightarrow 3^{\prime}\right)$ & Size (bp) \\
\hline Stk11 & $\begin{array}{l}\text { F: GGGCAACCTGCTACTCACC } \\
\text { R: CCAGATGTCCACCTTGAAAC }\end{array}$ & 103 \\
\hline p53 & $\begin{array}{l}\text { F: ATGAACCGCCGACCTATC } \\
\text { R: AGGGCAGGCACAAACACG }\end{array}$ & 98 \\
\hline$p 21$ & $\begin{array}{l}\text { F: GCCTTGTCGCTGTCTTGC } \\
\text { R: GCTGGTCTGCCTCCGTTTT }\end{array}$ & 95 \\
\hline $18 S$ rRNA & $\begin{array}{l}\text { F: AGGGGAGAGCGGGTAAGAGA } \\
\text { R: GGACAGGACTAGGCGGAACA }\end{array}$ & 241 \\
\hline
\end{tabular}

F, forward; R, reverse.

Animal Research Center, Shanghai First People's Hospital of Shanghai JiaoTong University (Shanghai, China). This study was approved by the Animal Ethics Committee of Shanghai JiaoTong University in compliance with the Experimental Animal Regulations of the National Science and Technology Commission, China (Permit no.SJTAEC201401). All mice were housed for 14 days, 3-4 per cage, in a temperature-controlled colony room under standard light-dark cycle conditions with access to food and water ad libitum. Cryoinjury was induced as previously described (7). In brief, the animals were divided into 2 groups: The untreated control group (6 animals not exposed to liquid nitrogen) and the cryoinjury experimental group (24 animals exposed to liquid nitrogen). A cryoprobe (Shanghai Qiujing Biochemical Reagent and Instrument Co. Ltd., Shanghai, China) with a diameter of $2.5 \mathrm{~mm}$ [similar in diameter to $\mathrm{C} 57 \mathrm{BL} / 6$ mouse corneas $(2.6 \mathrm{~mm})]$ was frozen in liquid nitrogen. First, the mice were intraperitoneally injected with $1.5 \%$ of $0.1 \mathrm{ml} / 20 \mathrm{~g}$ nembutal (Sigma-Aldrich, St. Louis, MO, USA). After anesthetizing, the cryoprobe was placed on the mouse cornea three times at 1 min intervals.

RNA extraction and analysis by reverse transcription-quantitative polymerase chain reaction (RT-qPCR). All steps were conducted as previously described (12). In brief, total cellular RNA was isolated using TRIzol reagent (Invitrogen Life Technologies, Carlsbad, CA, USA) according to the manufacturer's instructions. Then, the RNA samples were reverse-transcribed into cDNA using the ReverTra Ace- $\alpha$ First Strand cDNA Synthesis kit (TOYOBO,Osaka,Japan). RT-qPCR was conducted using a RealPlex4 real-time PCR detection system from Eppendorf Co. Ltd. (Hamburg, Germany), with SYBR Green RealTime PCR Master mix and detection dye (TOYOBO). RT-qPCR amplification was performed using the following steps: Denaturation at $95^{\circ} \mathrm{C}$ for $120 \mathrm{sec}$; followed by 40 cycles of denaturation at $95^{\circ} \mathrm{C}$ for $15 \mathrm{sec}$, annealing at $58^{\circ} \mathrm{C}$ for $45 \mathrm{sec}$, and extension at $72^{\circ} \mathrm{C}$ for $42 \mathrm{sec}$. Target cDNA was quantified using the relative quantification method. A comparative threshold cycle $(\mathrm{Ct})$ was used to determine gene expression relative to a control (calibrator) and steady-state mRNA levels are reported as an n-fold difference relative to the calibrator. For each sample, the marker gene $\mathrm{Ct}$ values were normalized using the formula $\Delta \mathrm{Ct}=\mathrm{Ct}$ _genes-Ct_18SrRNA.
To determine relative expression levels, the following formula was used: $\Delta \Delta \mathrm{Ct}=\Delta \mathrm{Ct}$ treated_group- $\Delta \mathrm{Ct}$ _control_group. The values used to the plot relative expression of markers were calculated using the expression $2^{-\Delta \Delta \mathrm{Ct}}$. The mRNA levels were calibrated based on levels of $18 \mathrm{~S}$ rRNA. The cDNA of each gene was amplified using primers as follows (Table I).

Histopathology. The cornea tissues were stained with hematoxylin and eosin (H\&E) for analysis by histopathology. Briefly, fresh tissues were washed three times with phosphate-buffered saline (PBS), fixed in 4\% paraformaldehyde (Sigma-Aldrich) for $30 \mathrm{~min}$, dehydrated through a graded series of ethanol, vitrified in xylene and embedded in paraffin. Next, 6- $\mu \mathrm{m}$ sections were cut in serial succession and stained with H\&E. The sections were analyzed using a microscope (DMI3000; Leica, Allendale, NJ, USA).

Immunohistochemistry. All steps were conducted as previously described (13). Briefly, fresh tissues were washed 3 times with PBS, fixed with $4 \%$ paraformaldehyde (Sigma-Aldrich) for $30 \mathrm{~min}$, dehydrated through a graded series of ethanol, vitrified in xylene and embedded in paraffin. Next, 6- $\mu \mathrm{m}$ sections were cut in serial succession, rinsed with $3 \%$ phosphate buffer, and underwent microwave heat repairing. Rabbit anti-mouse Stk11 polyclonal antibody (cat. no. sc-28788; Santa Cruz Biotechnology Inc., Santa Cruz, CA, USA; dilution, 1:100); rabbit anti-mouse p53 Ser15-pho polyclonal antibody (cat. no. sc-101762; Santa Cruz Biotechnology Inc.; dilution, 1:100); rabbit anti-mouse p21 polyclonal antibody (cat. no. sc-397; Santa Cruz Biotechnology Inc.; dilution, 1:100); and rabbit anti-mouse active caspase-3 polyclonal antibody (cat. no. 9661S; Cell Signaling Technology Inc., Danvers, MA, USA; 1:200) were added and incubated for $45 \mathrm{~min}$, followed by incubation with horseradish peroxidase-conjugated secondary antibody (cat. no. sc-2004; Santa Cruz Biotechnology Inc.; dilution, 1:100). Antibody detection was achieved with a color reaction using an $\mathrm{ABC}$ chromogenic reagent (Sigma-Aldrich). PBS ( $\mathrm{pH}$ 7.4) was used in the place of primary antibody as a negative control. Five randomly selected fields (x200 magnification) from each tissue section were observed and analyzed by Image-Pro Plus 6.0 software (Media Cybernetics Co. Ltd., Rochville, MD, USA). 


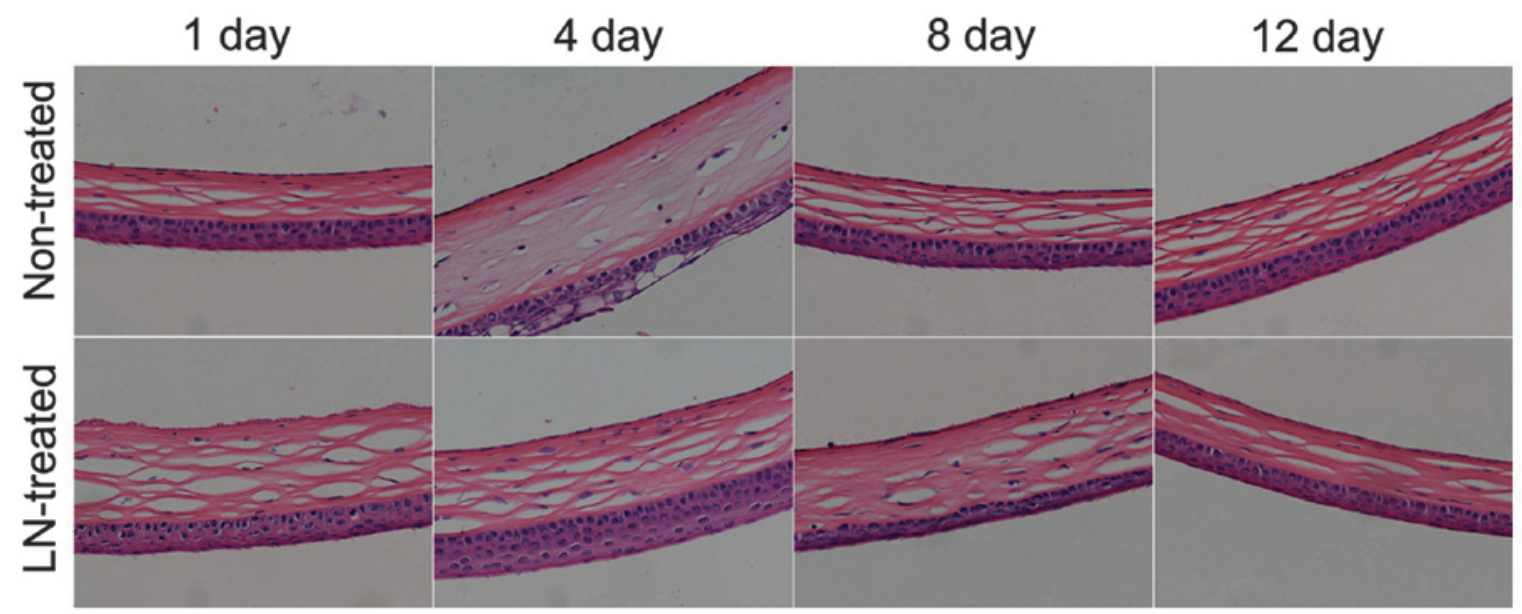

Figure 1. Pathology of corneal tissue in mice following cryoinjury. Corneal tissue was stained with hematoxylin and eosin to assess damage following cryoinjury (LN-treated) or no treatment (non-treated). Original magnification, x200. LN, liquid nitrogen.
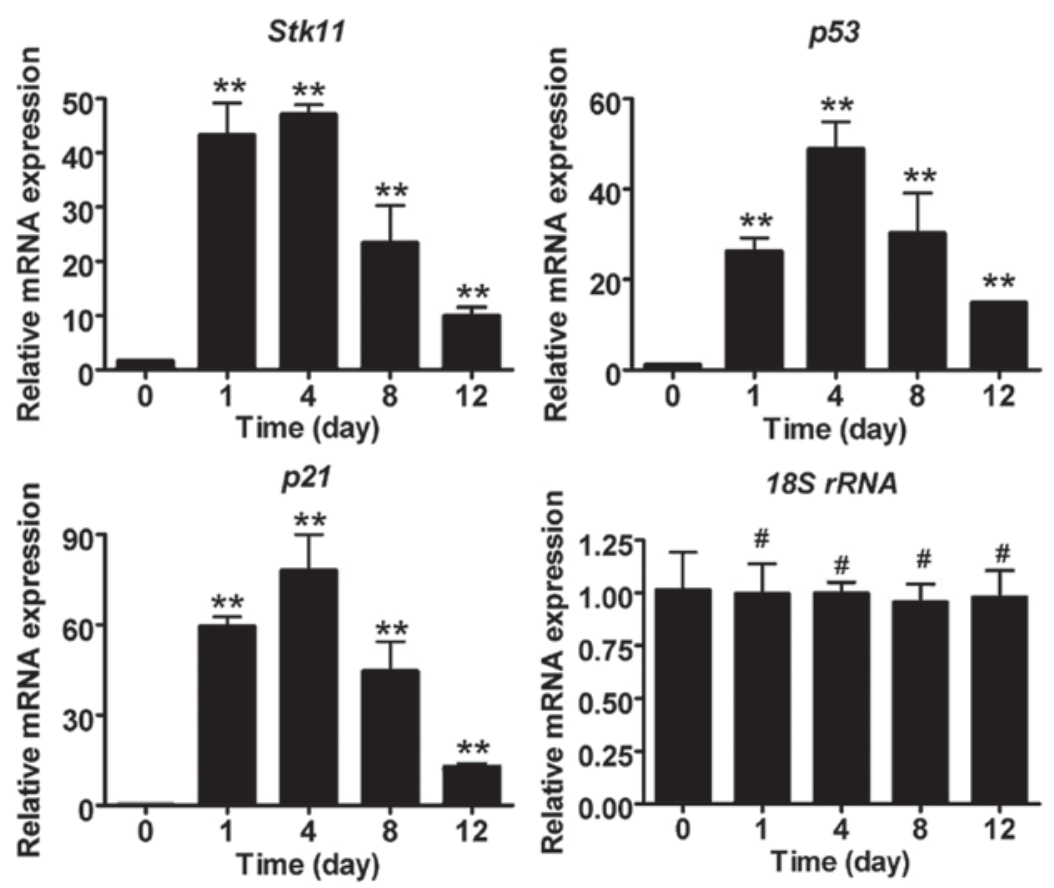

Figure 2. mRNA expression analysis of the Stk11-p53 signaling pathway by reverse transcription-quantitative polymerase chain reaction. Results indicated that mRNA expression levels of Stk11, p53 and $p 21$ (Stk11-p53 signal pathway factors) following cryoinjury were elevated compared with those at day 0 ( ${ }^{* *} \mathrm{P}<0.01$ and ${ }^{*} \mathrm{P}>0.05$ vs. 0 day; $\mathrm{n}=3$ ).

Chromatin immunoprecipitation (ChIP) assays. To perform ChIP experiments primary antibodies as used for IH and normal rabbit IgG (Upstate Biotechnology, Lake Placid, NY, USA) as a negative control were used. In brief, all steps were conducted as previously described (9). Cells were fixed in $1 \%$ formaldehyde for $30 \mathrm{~min}$ at $37^{\circ} \mathrm{C}$ and then quenched with $125-\mathrm{mM}$ glycine (Sigma-Aldrich). for $10 \mathrm{~min}$ at room temperature to create DNA-protein cross-links. Samples were sonicated on ice until chromatin fragments became 200-1,000 bp in size and were then incubated with antibodies at $4^{\circ} \mathrm{C}$ overnight. PCR amplification was performed under the following conditions: 33 cycles run by denaturation at $95^{\circ} \mathrm{C}$ for $30 \mathrm{sec}$, annealing at $55^{\circ} \mathrm{C}$ for $30 \mathrm{sec}$ and extension at $72^{\circ} \mathrm{C}$ for $30 \mathrm{sec}$.
Statistical analysis. Each experiment was performed at least three times. Data are shown as the mean \pm standard error and analyzed by Student's t-test when appropriate. $\mathrm{P}<0.05$ was considered to indicate a statistically significant difference. GraphPad Prism 5.00 (GraphPad Software Inc., La Jolla, CA, USA) was used for statistical analysis.

\section{Results}

Liquid nitrogen freezing causes significant damage to the mouse CECs. The healthy mouse CECs are round or polygonal, of similar size and tightly aligned. After low-temperature freezing with liquid nitrogen, injury to the corneal tissues and CECs was significant. First, the corneal tissues were observed 
Table II. Reverse transcription-quantitative polymerase chain reaction results.

\begin{tabular}{lccrrr}
\hline & \multicolumn{5}{c}{ Time (days) after liquid nitrogen freezing treatment } \\
\cline { 2 - 6 } Gene & 0 (non-treated) & 1 & 4 & 8 & 12 \\
\hline Stk11 & $1.627 \pm 0.094$ & $43.327 \pm 5.853$ & $47.048 \pm 1.793$ & $23.419 \pm 6.855$ & $9.888 \pm 1.663$ \\
p21 & $0.560 \pm 0.034$ & $59.519 \pm 3.164$ & $78.071 \pm 11.814$ & $44.564 \pm 9.789$ & $12.848 \pm 0.889$ \\
p53 & $1.102 \pm 0.003$ & $26.253 \pm 2.990$ & $48.881 \pm 6.067$ & $30.266 \pm 8.859$ & $14.827 \pm 0.206$ \\
18S rRNA & $1.016 \pm 0.178$ & $0.996 \pm 0.143$ & $0.998 \pm 0.052$ & $0.953 \pm 0.089$ & $0.976 \pm 0.129$ \\
\hline
\end{tabular}

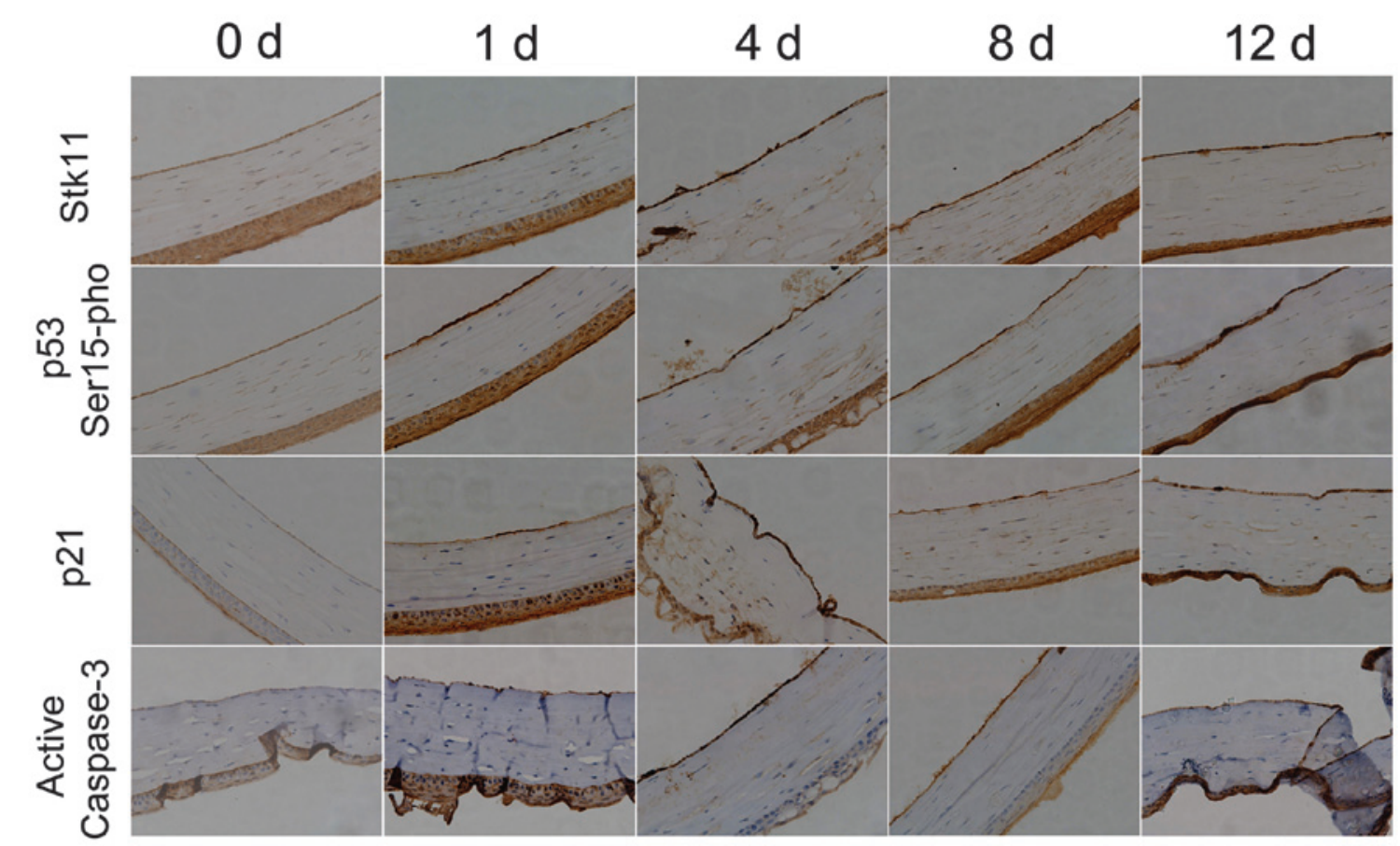

Figure 3. Expression analysis of Stk11-p53 signaling pathway proteins by immunohistochemistry. Staining of Stk11, p53 and p21 (Stk11-p53 signal pathway factors) strongly indicated induced expression following cryoinjury at several time-points. Original magnification: x200.

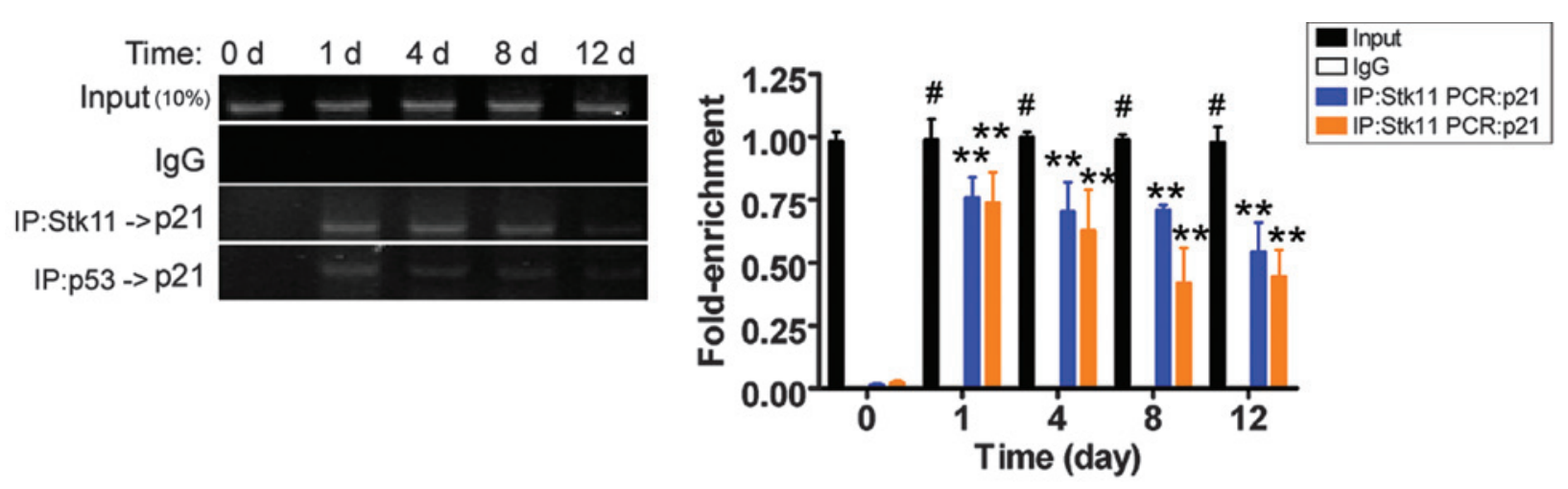

Figure 4. ChIP analysis of Stk11-p53 occupancy of the p21 gene promoter. The ChIP assay shows significant gene amplification bands using specific primers for the $p 21$ gene promoter on the 1st, 4th, and 8th day after cryoinjury, and weak amplification bands were observed on the 12th day after cryoinjury ( ${ }^{* *} \mathrm{P}<0.01$ and $" \mathrm{P}>0.05$ vs. 0 day; $\mathrm{n}=3$ ).

to be swollen; and CECs within the tissue were observed to swell, fragment and shed (Fig. 1). By the 12th day after liquid nitrogen treatment, CEC damage was partly repaired, and a number of new CECs were identified.
Liquid nitrogen freezing stimulates significant expression of Stk11-p53 signaling pathway components. To determine whether the expression of Stk11-p53 signal pathway components is induced in mouse CECs by liquid nitrogen freezing, 
RT-qPCR and IHC analysis were conducted. The mRNA expression levels of core Stk11-p53 signaling factors (Stk11, $p 53$ and $p 21)$ in mouse CECs were significantly elevated after cryoinjury compared with the untreated group (day 0) (Fig. 2; Table II). In addition, IHC confirmed that protein expression of Stk11, p53 and p21 was elevated, and caspase-3 was activated following cryoinjury (Fig. 3).

Cryoinjury stimulates 221 gene transcription. Previously, the Stk11-p53 complex was shown to specifically bind to the $p 21$ gene promoter (9). The results of the ChIP-PCR assay revealed that PCR amplification bands of the $p 21$ gene promoter were weaker on the 12th day after liquid nitrogen treatment. However, the PCR signals were not present in the untreated group (Fig. 4). These results suggest that cryoinjury induced Stk11-p53-mediated transcription of p21, which may eventually induce apoptosis.

\section{Discussion}

Extremely low temperatures can lead to corneal injury, and damage to CECs can affect vision $(7,14)$. Very brief contact with cold material can be sufficient to cause frostbite. Furthermore, in addition to possibly disrupting vision, cryoinjuries have relatively long recovery periods. Although the outcome of cryoinjury to CECs may be obvious, the mechanism of injury is not clear. In this study, the Stk11-p53 signaling pathway was observed to be involved in the mechanism of CEC cryoinjury based on the results of previous studies (7-9). These studies indicated that the Stk11-p53 signal pathway regulated cell proliferation (8-11). Usually, the Stk11-p53 signaling pathway is silenced during cell division and proliferation or in a stem cell state. However, when normal cells are exposed to external stimuli (such as oxidative damage), the Stk11-p53 signaling pathway is activated (8-11). When the Stk11-p53 signaling pathway is activated, it inhibits cell cycle progression, arresting cells at the G0/G1 phase to inhibit cell mitosis and ultimately resulting in apoptosis (8-11). Stk11 is a serine/threonine kinase, which regulates numerous physiological and pathological processes. The $S t k 11$ gene is generally considered to be a tumor suppressor gene (8-11). In this study, it was demonstrated that the Stk11-p53 signaling pathway was abnormally activated during mouse CEC cryoinjury. Four days following cryoinjury, the damage to CECs was significant and included swelling, partial necrosis and shedding. Simultaneously, the expression of the Stk11-p53 signaling pathway core factors (Stk11, p53, and p21) was significantly elevated. This suggested that the Stk11-p53 signaling pathway was involved in the apoptosis of mouse CECs following cryoinjury. Further investigation demonstrated found that cryoinjury damages mouse CECs and that Stk11 catalytically modified p53 by phosphorylation at serine residue 15 , which enhanced p53 activity. p53 activation resulted in specific binding to the $p 21$ gene promoter, and ultimately enhanced the transcriptional activity of the $p 21$ gene. In conclusion, extremely low temperatures lead to cryoinjury and apoptosis of mouse CECs due to Stk11-p53 signaling pathway activation. In conclusion, the Stk11-p53 signaling pathway may be a novel target for the treatment of corneal endothelial cell damage.

\section{Acknowledgements}

This study was supported by grant from National Natural Science Foundation of China (grant nos. 81371068 and 81202811) to Professor Yan Liu and Professor Te Liu.

\section{References}

1. Mimura T, Yamagami S and Amano S: Corneal endothelial regeneration and tissue engineering. Prog Retin Eye Res 35: 1-17, 2013.

2. Williams KA, Irani YD and Klebe S: Novel therapeutic approaches for corneal disease. Discov Med 15: 291-299, 2013.

3. Joyce NC: Proliferative capacity of corneal endothelial cells. Exp Eye Res 95: 16-23, 2012.

4. Lam FC, Bruinsma M and Melles GR: Descemet membrane endothelial transfer. Curr Opin Ophthalmol 25: 353-357, 2014.

5. Peh GS, Beuerman RW, Colman A, Tan DT and Mehta JS: Human corneal endothelial cell expansion for corneal endothelium transplantation: An overview. Transplantation 91: 811-819, 2011.

6. Hayashi T, Yamagami S, Tanaka K, Yokoo S, Usui T, Amano S and Mizuki N: A mouse model of allogeneic corneal endothelial cell transplantation. Cornea 27: 699-705, 2008.

7. Han SB, Ang H, Balehosur D, Peh G, Chaurasia SS, Tan DT and Mehta JS: A mouse model of corneal endothelial decompensation using cryoinjury. Mol Vis 19: 1222-1230, 2013.

8. Liang X, Wang P, Gao Q, Xiang T and Tao X: Endogenous LKB1 knockdown accelerates $\mathrm{G}(1) / \mathrm{S}$ transition through p53 and p16 pathways. Cancer Biol Ther 9: 156-160, 2010.

9. Zeng PY and Berger SL: LKB1 is recruited to the p21/WAF1 promoter by $\mathrm{p} 53$ to mediate transcriptional activation. Cancer Res 66: 10701-10708, 2006.

10. Vaahtomeri K and Mäkelä TP: Molecular mechanisms of tumor suppression by LKB1. FEBS Lett 585: 944-951, 2011.

11. Krock B, Skuli N and Simon MC: The tumor suppressor LKB1 emerges as a critical factor in hematopoietic stem cell biology. Cell Metab 13: 8-10, 2011.

12. Liu T, Chen Q, Huang Y, Huang Q, Jiang L and Guo L: Low microRNA-199a expression in human amniotic epithelial cell feeder layers maintains human-induced pluripotent stem cell pluripotency via increased leukemia inhibitory factor expression. Acta Biochim Biophys Sin (Shanghai) 44: 197-206, 2012.

13. Shen DZ, Xin SL, Chen C and Liu T: Effect of atorvastatin on expression of TLR4 and NF- $\mathrm{KB}$ p65 in atherosclerotic rabbits. Asian Pac J Trop Med 6: 493-496, 2013.

14. Liu H, Zhang J, Liu CY, Hayashi Y and Kao WW: Bone marrow mesenchymal stem cells can differentiate and assume corneal keratocyte phenotype. J Cell Mol Med 16: 1114-1124, 2012. 